\title{
Avaliação da composição corporal dos pacientes em ambulatório de nutrição
}

\author{
Evaluation of the body composition of patients \\ in a nutrition outpatient clinic
}

\begin{abstract}
RESUMO A avaliação da composição corporal possibilita verificar desequilíbrios entre a quantidade de gordura e musculatura, quadro atrelado a alterações metabólicas. O objetivo do estudo foi considerar a composição corporal com enfoque para a massa muscular. Tratou-se de um estudo transversal, com indivíduos de 18 a 60 anos de idade. Aplicou-se o teste T-Student para amostras independentes para: idade, massa muscular ( $\mathrm{MM} \mathrm{Kg})$, porcentagem de massa muscular (\%MM), índice de massa muscular (IMM), circunferência da cintura (CC) e índice de massa corporal (IMC), e estratificados entre sarcopênicos e não sarcopênicos e a correlação linear de Pearson entre CC e IMC com variáveis de massa muscular. Os dados foram calculados pelo programa Stat versão 6.0 e o nível de significância adotado $\mathrm{p}<0,05$. Averiguou-se uma média de idade de $34,08 \pm 9,5$ anos, com predomínio de mulheres e $63,83 \%$ dos indivíduos praticavam exercício. Houve diferença significativa no comparativo entre gêneros para CC, MM, \%MM e IMM e entre homens sarcopênicos e não sarcopênicos, a diferença foi significativa para idade, CC, MM (Kg), IMM e IMC. Foi verificada uma correlação negativa entre a CC com MM, \% MM em homens, mulheres e para a amostra geral ( $\mathrm{p}$ $<0,05)$. Haja vista os resultados, há um comprometimento da musculatura esquelética em razão do excesso de peso corporal, sobretudo sarcopenia em homens, desencadeada em idades precoces.

Palavras-chave: Composição corporal. Antropometria. Sarcopenia. Obesidade. Doenças crônicas.
\end{abstract}

Beatriz Cassaniga Talassi ${ }^{1}$ AMANDA MARTim ${ }^{1}$ Iza Oliveira HoFf ${ }^{I}$

${ }^{\mathrm{I}}$ Universidade Metodista de Piracicaba (UNIMEP). Piracicaba/ SP - Brasil.
Aвstract The evaluation of the body composition allows to verify imbalances between the amount of fat and musculature, a framework linked to metabolic alterations. The objective of the study was to evaluate the body composition with a focus on muscle mass. It was a cross-sectional study with individuals aged 18-60 years. The T-Student test was used for independent samples for age, muscle mass (MM Kg), percentage of muscle mass (\% MM), muscle mass index (IMM), waist circumference (CC) and body mass index (IMC), and stratified between sarcopenic and non-sarcopenic, and Pearson's linear correlation between CC and IMC with muscle mass variables. The data were calculated by the Stat program version 6.0 and the level of significance adopted $p<0,05$. A mean age of $34,08 \pm 9,5$ years was found, with a predominance of women and $63.83 \%$ of the individuals practicing exercise. There was a significant difference between the genders for CC, MM, $\% \mathrm{MM}$ and IMM and between sarcopenic and non sarcopenic men, the difference was significant for age, CC, MM (Kg), IMM and IMC. A negative correlation was found between CC with MM, MM\% in men, women and for the general sample $(p<0,05)$. Given the results, there is a compromise of the skeletal muscles due to excess body weight, especially sarcopenia in men, triggered at early ages.

Keywords: Body composition. Anthropometry. Sarcopenia. Obesity. Chronic diseases. 


\section{INTRODUÇÃO}

O desequilíbrio da composição corporal (massa livre de gordura e massa gorda) pode estar relacionado à inadequação dos hábitos alimentares, sobretudo à ingestão proteica insuficiente e o excesso calórico advindo de carboidratos simples e lipídios, que contribuem para o decréscimo de massa muscular (sarcopenia) e/ou acúmulo adiposo em quadros isolados ou conjuntamente. ${ }^{1}$

$\mathrm{O}$ excesso de tecido adiposo impulsiona o depósito de ácidos graxos ao redor e no interior das células musculares, à medida que células satélites do tecido muscular esquelético passam a adquirir as características inerentes a um adipócito maduro. ${ }^{2}$ Ademais, há maior secreção de IL-6 e TNF- $\alpha$ nos adipócitos da região abdominal, que exercem maior ação proteolítica do que a própria gordura subcutânea $^{3}$ e, por conseguinte, ocorre a redução da qualidade muscular, levando a prejuízos na capacidade de síntese proteica. Logo, a obesidade acabaria impulsionando a perda de massa muscular. ${ }^{4}$

Estudo com indivíduos em idade superior a 20 anos verificou que a obesidade sarcopênica demonstrou forte associação com resistência à insulina, alterações glicêmicas, pré-diabetes e diabetes, predispondo ao desenvolvimento de complicações metabólicas mais acentuadas do que quando se considera sarcopenia ou obesidade isoladamente. ${ }^{5}$ Como a massa muscular tem ação protetiva às doenças cardiovasculares, quando há déficit muscular, sobretudo com concomitante aumento da adiposidade corporal, contribui-se para o desencadeamento de desordens cardiovasculares. ${ }^{6}$ Obesos sarcopênicos, em comparação a indivíduos saudáveis, têm chances seis vezes superiores a ter uma maior pressão arterial. ${ }^{7}$

Para a avaliação antropométrica, deve-se lançar mão de índices direcionados à análise da musculatura, entre os quais pode-se citar o índice de massa muscular, ${ }^{8}$ que quando inferior ao padrão estabelecido para a média da população de referência, caracteriza o quadro de sarcopenia. Adicionalmente, quando há alteração da circunferência da cintura e/ou percentual de gordura corporal, pode-se diagnosticar a obesidade sarcopênica, ${ }^{1}$ prevenindo as limitações funcionais a que são expostos os indivíduos em diagnóstico tardio.

Haja vista a temática exposta, o presente estudo objetivou por meio da aferição antropométrica, a avaliação corporal, incluindo a análise de massa muscular dos pacientes atendidos no ambulatório de nutrição clínica. Ademais, verificar se o excesso de peso corporal estaria relacionado ao comprometimento quantitativo da musculatura esquelética.

\section{INDIVÍDUOS E MÉTODOS}

A avaliação foi composta por 53 pacientes de ambos os gêneros, com idade acima de 18 anos e menores de 60 anos. Como critérios de inclusão, somente os pacientes que foram atendidos na clínica de nutrição da Universidade Metodista de Piracicaba e que possuíssem todos os membros. Como exclusão, se enquadraram aqueles que faziam uso de drogas ilícitas, pacientes amputados, os que possuíam deficiência física e/ou mental, indivíduos acima de 60 anos e menores de 18 anos de idade e os pacientes que durante a avaliação apresentaram insta- 
bilidade do adipômetro e caso possuíssem alguma doença previamente diagnosticada, capaz de interferir nos resultados da avaliação. Ao consentirem participar, assinaram o Termo de Consentimento Livre e Esclarecido. O projeto foi aprovado pelo Comitê de Ética em Pesquisa - UNIMEP, sob o protocolo $\mathrm{n}^{\circ}$. 26/2016.

A primeira parte do estudo correspondeu à aquisição de informações referentes aos dados demográficos: nome, gênero, data de nascimento, idade e etnia; a anamnese clínica e nutricional: motivo da consulta, utilização de medicamentos e/ou suplementos (descrição do tipo e horário), prática de atividade física, que foi considerada quando realizada no mínimo três vezes/semana durante $20 \mathrm{mi}$ nutos, de acordo com o International Physical Activity Questionnaire ${ }^{9}$ e hábito de fumar e ingestão de bebidas alcoólicas (considerados quando referidos na frequência mínima de duas vezes semanais) e em quantidades significativas para riscos de saúde, correspondente a duas doses diárias conforme proposto pela Organization World Health. ${ }^{10}$ Foi feita a avaliação dos antecedentes familiares por meio do questionamento da presença de doenças crônicas: obesidade, hipertensão arterial sistêmica, diabetes mellitus e câncer em familiares próximos (pai, mãe, tios, avós, avôs e irmãos para ambos os antecedentes paterno e materno).

Os pacientes foram submetidos à avaliação do peso corporal, aferido por balança digital tipo plataforma Filizola ${ }^{\circ}$, com capacidade de até $300 \mathrm{Kg}$ e precisão de 0,1 $\mathrm{Kg}$. A medida foi realizada com os pacientes utilizando o mínimo de roupa possível, descalços, conforme proposto por Heyward e Stolarczyk. ${ }^{11}$ Após estabilizado o valor re- sultante da pesagem, esse foi registrado em quilogramas. ${ }^{12} \mathrm{~A}$ estatura foi mensurada por estadiômetro Filizola fixo à parede, com precisão de $0,1 \mathrm{~cm}$. Os indivíduos foram posicionados junto à superfície vertical plana do estadiômetro, tocando em cinco pontos do corpo (calcanhares, panturrilha, nádegas, ombros e cabeça), respeitando-se o plano horizontal de Frankfurt. Em seguida, registrou-se a altura em centímetros, segundo protocolo de Lohman, Roche e Martorell. ${ }^{13}$

Para a avaliação da massa muscular, foram realizadas aferições das dobras cutâneas em milímetros, utilizando-se o adipômetro (Lange $\left.{ }^{\circledR}\right)$ : triciptal (DCT), coxa (DCC) e panturrilha (DCP) e para as circunferências em centímetros (fita milimétrica de metal inextensível e inelástica, com precisão de $0,5 \mathrm{~cm}$ ): braço $(\mathrm{CB})$, coxa $(\mathrm{CCx})$, panturrilha (CP) e cintura (CC). Ambas, dobras e circunferências, foram obtidas de acordo com a padronização proposta por Lohman, Roche e Martorell, ${ }^{13}$ sendo as dobras cutâneas feitas em triplicata.

Com os dados peso e altura dos pacientes, foi estabelecido o índice de massa corporal (IMC), obtido pela razão entre o peso corporal expresso em quilogramas $(\mathrm{Kg})$ e a estatura em metros quadrados $\left(\mathrm{m}^{2}\right)$ e classificado conforme o parâmetro universal para a classificação de adultos acima de 20 anos de idade. ${ }^{14}$ Os indivíduos foram classificados quanto aos riscos de complicações metabólicas associadas à obesidade em função da circunferência da cintura, mensurada no ponto de menor circunferência, geralmente situada entre o último arco intercostal ou quando não identificada essa circunferência visualmente, adotou-se a aferição do ponto médio entre o último arco intercostal e a 
crista ilíaca, ${ }^{11}$ considerando-se alterados os resultados definidos pelo Consenso Latino-americano de Obesidade: ${ }^{15}$ aumentada (80 $\mathrm{cm}$ e $94 \mathrm{~cm}$ para mulheres e homens, respectivamente) e muito aumentada em $88 \mathrm{~cm}$ para mulheres e $102 \mathrm{~cm}$ para os homens.

$O$ valor de referência para a circunferência da panturrilha (CP) foi de $31 \mathrm{~cm}$ utilizado para a identificação da musculatura, os resultados inferiores a $31 \mathrm{~cm}$ foram classificados como sarcopenia. ${ }^{16}$ Acerca da circunferência muscular do braço (CMB), foi calculada segundo: $\mathrm{CMB}(\mathrm{cm})=\mathrm{CB}(\mathrm{cm})-\left[\pi^{*}\right.$ $x \operatorname{DCT}(\mathrm{cm})]$, no qual o valor de $\pi$ equivale a 3,14. A classificação adotada foi proposta por Frisancho. ${ }^{17}$

O cálculo da massa muscular (MM Kg) foi obtido pela equação de Lee et al. ${ }^{18} \mathrm{O}$ percentual de massa muscular, em relação à massa corporal, estabelecido como indicativo de sarcopenia: $\leq 27 \%$ para mulheres e $\leq 38 \%$ para homens. ${ }^{19}$ A partir do resultado da massa muscular (Kg), esses indivíduos foram classificados quanto ao grau de sarcopenia, pelo índice de massa muscular (IMM), com a equação de Baumgartner et al., ${ }^{20}$ cujo IMM $\left(\mathrm{Kg} / \mathrm{m}^{2}\right)=\mathrm{MM}(\mathrm{Kg}) /$ estatura $^{2}$ e a classificação em sarcopenia grau I: quando $\mathrm{IMM}>5,75 \mathrm{e} \leq 6,75 \mathrm{Kg} / \mathrm{m}^{2}$ e $>8,5$ $\mathrm{e} \leq 10,75 \mathrm{Kg} / \mathrm{m}^{2}$ para mulheres e homens, respectivamente, e a sarcopenia grau II: $\mathrm{mu}$ lheres $\mathrm{IMM} \leq 5,75 \mathrm{Kg} / \mathrm{m}^{2}$ e $\leq 8,5 \mathrm{Kg} / \mathrm{m}^{2}$ para homens. ${ }^{8}$

Foi realizado o teste T-Student para amostras independentes por grupo, considerando-se a averiguação de variáveis: idade, massa muscular (MM Kg, MM \% e IMM) e excesso de peso corporal (CC e IMC), sendo verificado os resultados dos mesmos, quando estratificado em indivíduos sarcopênicos e não sarcopênicos (de acordo com o IMM) e a correlação linear de Pearson entre CC e IMC com variáveis correspondentes à massa muscular (MM Kg, \% MM e IMM). Os dados foram calculados pelo programa Stat versão 6.0, sendo adotado como nível de significância o valor de $p<0,05$.

\section{RESUltados}

Entre os 53 indivíduos avaliados houve a exclusão de seis pacientes, uma por doença autoimune, que fazia uso de medicamentos que poderiam superestimar os resultados da avaliação corporal, uma paciente com IMC maior que $40 \mathrm{Kg} / \mathrm{m}^{2}$, no qual houve instabilidade do adipômetro, e outros quatro pacientes por não apresentarem vestimenta adequada no dia da avaliação, impossibilitando a aferição das medidas na primeira consulta e esses não retornaram ao ambulatório no dia agendado para um novo atendimento. Do total final composto por 47 voluntários, $77,4 \%(n=41)$ eram do gênero feminino e $22,6 \%(n=12)$ pertencentes ao gênero masculino, com média de idade 34,08 $\pm 9,5$ anos. Ao verificar a etnia dos pacientes, 39 eram brancos e 8 negros, não houve paciente asiático.

Quanto ao relato do hábito de fumar, obteve-se em 2,13\% ( $n=1)$ sendo do gênero feminino. Em contrapartida, 23,4\% $(n=11)$ distribuídos em $22,85 \%(n=8)$ para o gênero feminino e $25 \%(n=3)$ para o masculino, frente a $76,6 \%(n=36)$ dos indivíduos, que não relataram hábito de ingestão alcoólica. Em relação à prática de atividade física, $63,83 \%(n=30)$ realizavam atividade física $(3 \mathrm{x} /$ semanais ou mais), enquanto que $36,17 \% \quad(n=17)$ eram sedentários ou não praticavam com regularidade. 
Ao analisar a doença de base dos pacientes, observou-se que $36,17 \% \quad(n=17)$ apresentavam uma ou mais doenças e houve uma maior prevalência de hipertensão arterial sistêmica $(n=10)$, seguida de dislipide$\operatorname{mia}(\mathrm{n}=4)$.

A hipertensão arterial sistêmica (HAS) foi a doença presente na história familiar da maioria dos indivíduos, sendo 21 para antecedentes paternos e 24 para antecedentes maternos, considerando a amostra como um todo. Observou-se uma associação entre o componente hereditário materno para o desenvolvimento da HAS, citado pelas mulheres 57,14\% $(n=20)$. Em relação aos homens, a HAS surge preponderantemente em antecedentes paternos $(n=8)$, correspondente a $66,66 \%$ dos homens.

A média de idade de 34,08 $\pm 9,5$ anos revela uma amostra jovem, porém que já apresenta um sobrepeso pelo IMC, com circunferência da cintura alterada em ambos os gêneros, sendo em mulheres a medida muito aumentada (superior a $88 \mathrm{~cm}$ ) e em homens aumentada (acima de $94 \mathrm{~cm}$ ), conforme a Tabela 1 .

Tabela 1 - Caracterização geral da amostra e por gênero referente aos dados antropométricos.

\begin{tabular}{l|c|c|c|c}
\hline Medidas aferidas & $\begin{array}{c}\text { Amostra }(\mathrm{n}=47) \\
\mathrm{M} \pm \mathrm{DP}\end{array}$ & $\begin{array}{c}\text { Mulheres } \\
(\mathrm{n}=35) \mathrm{M} \pm \mathrm{DP}\end{array}$ & $\begin{array}{c}\text { Homens } \\
(\mathrm{n}=12) \mathrm{M} \pm \mathrm{DP}\end{array}$ \\
\hline Idade $($ anos $)$ & $34,08 \pm 9,5$ & $33,65 \pm 9,21$ & $35,33 \pm 10,64$ & 0,603 \\
\hline Peso $(\mathrm{Kg})$ & $81,5 \pm 16,25$ & $78,39 \pm 14,33$ & $90,57 \pm 18,68$ & 0,056 \\
\hline Estatura $(\mathrm{m})$ & $1,66 \pm 0,09$ & $1,62 \pm 0,06$ & $1,77 \pm 0,08$ & $0,00008^{*}$ \\
\hline $\mathrm{DCP}(\mathrm{mm})$ & $29,82 \pm 14,06$ & $33,67 \pm 13,03$ & $18,58 \pm 10,84$ & $0,001^{*}$ \\
\hline $\mathrm{CP}(\mathrm{cm})$ & $39,92 \pm 3,62$ & $39,74 \pm 3,76$ & $40,46 \pm 3,28$ & 0,56 \\
\hline $\mathrm{CC}(\mathrm{cm})$ & $93,74 \pm 12,93$ & $91,17 \pm 11,02$ & $101,25 \pm 15,51$ & $0,018^{*}$ \\
\hline $\mathrm{MM}(\mathrm{Kg})$ & $24,35 \pm 6,19$ & $21,89 \pm 4,34$ & $31,53 \pm 5,13$ & $0,000^{*}$ \\
\hline $\mathrm{MM}(\%)$ & $30,34 \pm 6,79$ & $28,5 \pm 5,83$ & $35,7 \pm 6,75$ & $0,0009^{*}$ \\
\hline $\mathrm{IMM}\left(\mathrm{Kg} / \mathrm{m}^{2}\right)$ & $8,78 \pm 1,76$ & $8,34 \pm 1,57$ & $10,04 \pm 1,72$ & $0,003^{*}$ \\
\hline $\mathrm{IMC}\left(\mathrm{Kg} / \mathrm{m}^{2}\right)$ & $29,52 \pm 5,15$ & $29,85 \pm 5,31$ & $28,57 \pm 4,73$ & 0,464 \\
\hline $\mathrm{CMB}(\mathrm{cm})$ & $23,43 \pm 4,26$ & $22,13 \pm 3,69$ & $27,23 \pm 3,56$ & $0,001^{*}$ \\
\hline
\end{tabular}

Valores descritos em média \pm desvio padrão. DCT: dobra cutânea tricipital; DCC: dobra cutânea da coxa; DCP: dobra cutânea da panturrilha; CP: circunferência da panturrilha; CC: circunferência da cintura; MM: massa muscular; IMM: índice de massa muscular; IMC: índice de massa corporal; CMB: circunferência muscular do braço; n: número de indivíduos. ${ }^{*}$ Valor de $p$ significativo, $<0,05$. 
Embora a CP tenha dado adequada, ela sofre com infiltração gordurosa, visto que houve diferença estatística na análise de DCP, que é mais específica para a avaliação de gordura localizada na região. Os resultados demonstraram que houve diferença estatística para a amostra geral, considerando-se a circunferência da cintura, massa muscular (Kg e \%) e IMM, cujos valores em homens denotam sarcopenia. A média do índice de massa muscular (IMM) para o sexo masculino $\left(10,04 \pm 1,72 \mathrm{Kg} / \mathrm{m}^{2}\right)$ indica sarcopenia grau I, enquanto que o resultado de $8,34 \pm 1,57 \mathrm{Kg} / \mathrm{m}^{2}$ das mulheres encontra-se dentro da normalidade. Os resultados do IMM vão ao encontro da sarcopenia identificada pelo \% MM em homens, uma vez que a média $35,7 \% \pm 6,75$ de massa muscular para homens é inferior à referência de $37 \% .{ }^{19}$

De acordo com a classificação de sarcopenia pelo índice de massa muscular, 25,53\% dos indivíduos voluntários apresentam sarcopenia, acometendo $66,67 \%$ dos homens $(n=8)$ e $11,43 \%(n=4)$ mulheres que seriam sarcopênicas. Para a amostra geral, $57,45 \%(n=27)$ são classificados como sarcopênicos, considerando a análise pelo \% de massa muscular, sendo 66,67\% $(n=8)$ dos homens e 54,29\% $(n=19)$ das mulheres. Por análise de \%MM e IMM, em ambos, obteve-se um resultado de $66,67 \%$ entre o gênero masculino que mostraram comprometimento da musculatura compatível com esse diagnóstico.

Entre o total de 25,53\% $(n=12)$ de indivíduos sarcopênicos, 66,66\% $(n=8)$ exibem obesidade sarcopênica, resultado correspondente a $17,03 \%$ da amostra total $(n=8)$ diagnosticada obesa sarcopênica, um percentual de $50 \%(n=4)$ para as mulheres e $50 \%$ para homens $(n=4)$. Considerando a amostra total, 17,03\% dos indivíduos são obesos sarcopênicos, visto que 33,33\% dos homens $(n=4)$ e $11,43 \%$ das mulheres $(n=4)$ apresentaram sarcopenia e alteração da circunferência abdominal concomitantemente, sendo o resultado evidenciado na tabela a seguir:

Tabela 2 - Diagnóstico de obesidade sarcopênica.

\begin{tabular}{l|c|c|c}
\hline Diagnóstico & Mulheres $(\mathrm{n}=35)$ & Homens $(\mathrm{n}=12)$ & Total $(\mathrm{n}=47)$ \\
\hline Sarcopenia (IMM) & $11,43 \%$ & $66,67 \%$ & $25,53 \%$ \\
\hline Alteração da CC & $85,72 \%$ & $66,66 \%$ & $80,86 \%$ \\
\hline Obesidade sarcopênica & $11,43 \%$ & $33,33 \%$ & $17,03 \%$ \\
\hline
\end{tabular}

Sobre os riscos de complicações metabólicas associadas à obesidade em função da circunferência da cintura, 68,09\% dos indivíduos, independentemente do gênero, apresentaram uma circunferência muito aumentada e $12,77 \%$ aumentada, podendo-se concluir que $80,86 \%$ mostraram alteração para essa medida em algum grau e considerando somente para o gênero feminino, esse resultado acaba sendo maior (85,72\%). Observou-se que $71,43 \%$ das pacientes tinham uma distribuição de gordura bastante acentuada na região abdominal, enquanto que entre os homens 33,33\% apresentaram uma circunferência dentro da normalidade e 58,33\% muito aumentada, conforme a tabela a seguir: 
Tabela 3 - Riscos de complicações cardiovasculares associadas à circunferência da cintura.

\begin{tabular}{l|c|c|c}
\hline Circunferência da cintura & Mulheres $(\mathrm{n}=35)$ & Homens $(\mathrm{n}=12)$ & Total $(\mathrm{n}=47)$ \\
\hline Normal & $14,29 \%$ & $33,33 \%$ & $19,15 \%$ \\
\hline Aumentado & $14,29 \%$ & $8,33 \%$ & $12,77 \%$ \\
\hline Muito aumentado & $71,43 \%$ & $58,33 \%$ & $68,09 \%$ \\
\hline
\end{tabular}

Em relação às variáveis de composição corporal relacionadas ao excesso de peso e comprometimento da massa muscular em homens, a idade apresentou diferença significativa entre o grupo sarcopênico e não sarcopênico, pois indivíduos sarcopênicos eram mais jovens do que aqueles que exibi- ram valores para massa muscular dentro da normalidade. Em relação IMC, aqueles que apresentavam sarcopenia tinham uma média de IMC de $26,19 \mathrm{Kg} / \mathrm{m}^{2}$, valor classificado em sobrepeso ${ }^{14}$ e possuíam uma circunferência da cintura aumentada ${ }^{15}(94,62 \pm 14,45)$, conforme descrito na Tabela 4.

Tabela 4 - Variáveis da composição corporal e idade entre homens sarcopênicos e não sarcopênicos.

\begin{tabular}{l|c|c|c}
\hline \multicolumn{1}{c|}{ Variáveis } & $\begin{array}{c}\text { Não sarcopênicos } \\
(\mathrm{n}=4) \mathrm{M} \pm \mathrm{DP}\end{array}$ & $\begin{array}{c}\text { Sarcopênicos } \\
(\mathrm{n}=8) \mathrm{M} \pm \mathrm{DP}\end{array}$ & Valor de $p$ \\
\hline Idade (anos) & $47,00 \pm 10,42$ & $28,5 \pm 3,85$ & $0,001^{*}$ \\
\hline $\mathrm{CC}(\mathrm{cm})$ & $114,5 \pm 6,66$ & $94,62 \pm 14,45$ & $0,027^{\star}$ \\
\hline $\mathrm{MM}(\mathrm{Kg})$ & $36,81 \pm 4,16$ & $28,89 \pm 3,18$ & $0,004^{*}$ \\
\hline $\mathrm{MM}(\%)$ & $35,65 \pm 1,16$ & $35,73 \pm 8,42$ & 0,985 \\
\hline $\mathrm{IMM}\left(\mathrm{Kg} / \mathrm{m}^{2}\right)$ & $11,89 \pm 0,91$ & $9,12 \pm 1,16$ & $0,002^{*}$ \\
\hline $\mathrm{IMC}\left(\mathrm{Kg} / \mathrm{m}^{2}\right)$ & $33,34 \pm 2,02$ & $26,19 \pm 3,72$ & $0,005^{*}$ \\
\hline
\end{tabular}

*Valor de $p$ significativo, $<0,05$.

Para o gênero feminino, de modo diferente aos homens, a sarcopenia não teve relação com idades jovens. Independentemente de apresentarem ou não sarcopenia, as mulheres tinham uma circunferência da cintura muito aumentada, ${ }^{15}$ embora sem diferença estatística (Tabela 5). 
Tabela 5 - Variáveis da composição corporal e idade entre mulheres sarcopênicas e não sarcopênicas.

\begin{tabular}{l|c|c|c}
\hline \multicolumn{1}{c|}{ Variáveis } & $\begin{array}{c}\text { Não sarcopênicas } \\
(\mathrm{n}=31) \mathrm{M} \pm \mathrm{DP}\end{array}$ & $\begin{array}{c}\text { Sarcopênicas } \\
(\mathrm{n}=4) \mathrm{M} \pm \mathrm{DP}\end{array}$ & Valor de $p$ \\
\hline Idade (anos) & $34,45 \pm 9,29$ & $27,5 \pm 6,45$ & 0,158 \\
\hline $\mathrm{CC}(\mathrm{cm})$ & $91,37 \pm 11,71$ & $89,62 \pm 1,7$ & 0,770 \\
\hline $\mathrm{MM}(\mathrm{Kg})$ & $22,39 \pm 4,36$ & $17,97 \pm 0,83$ & 0,054 \\
\hline $\mathrm{MM}(\%)$ & $29,13 \pm 5,83$ & $23,66 \pm 3,17$ & 0,077 \\
\hline $\mathrm{IMM}\left(\mathrm{Kg} / \mathrm{m}^{2}\right)$ & $8,6 \pm 1,47$ & $6,31 \pm 0,27$ & $0,004^{*}$ \\
\hline $\mathrm{IMC}\left(\mathrm{Kg} / \mathrm{m}^{2}\right)$ & $30,22 \pm 5,46$ & $26,95 \pm 3,02$ & 0,252 \\
\hline
\end{tabular}

*Valor de $p$ significativo, $<0,05$.

Avaliando-se a relação existente entre a musculatura e a distribuição de gordura corporal, que caracteriza a obesidade sarcopênica, foi verificada a existência de uma correlação negativa significativa para a amostra geral e por gênero para \% MM. A massa muscular em $\mathrm{Kg}$ somente apresentou resultado significativo para a amostra geral. Os resultados estão descritos na Tabela 6 .

Tabela 6 - Correlação entre circunferência da cintura e variáveis referentes à massa muscular.

\begin{tabular}{l|c|c|c}
\hline $\begin{array}{l}\text { Circunferência da } \\
\text { cintura }\end{array}$ & MM $(\mathrm{Kg})$ & $\% \mathrm{MM}$ & $\mathrm{IMM}\left(\mathrm{Kg} / \mathrm{m}^{2}\right)$ \\
\hline Geral & $\mathrm{r}=0,42(p=0,04)^{\star}$ & $\mathrm{r}=-0,41(p=0,004)^{\star}$ & $\mathrm{r}=0,27(p=0,06)$ \\
\hline Homens & $\mathrm{r}=0,11(p=0,54)$ & $\mathrm{r}=-0,72(p=0,000)^{\star}$ & $\mathrm{r}=0,070(p=0,70)$ \\
\hline Mulheres & $\mathrm{r}=0,56(p=0,06)$ & $\mathrm{r}=-0,66(p=0,02)^{\star}$ & $\mathrm{r}=0,32(p=0,32)$ \\
\hline
\end{tabular}

r: coeficiente de correlação linear. ${ }^{\star}$ Valor de $p$ significativo, $<0,05$.

O gráfico a seguir enfatiza especificamente a circunferência da cintura e \% MM. Nota-se forte correlação entre as variáveis, especialmente entre o gênero masculino $(-0,72)$, com $p<0,001$. 
Gráfico 1 - Correlação entre circunferência da cintura e percentual de massa muscular em homens.

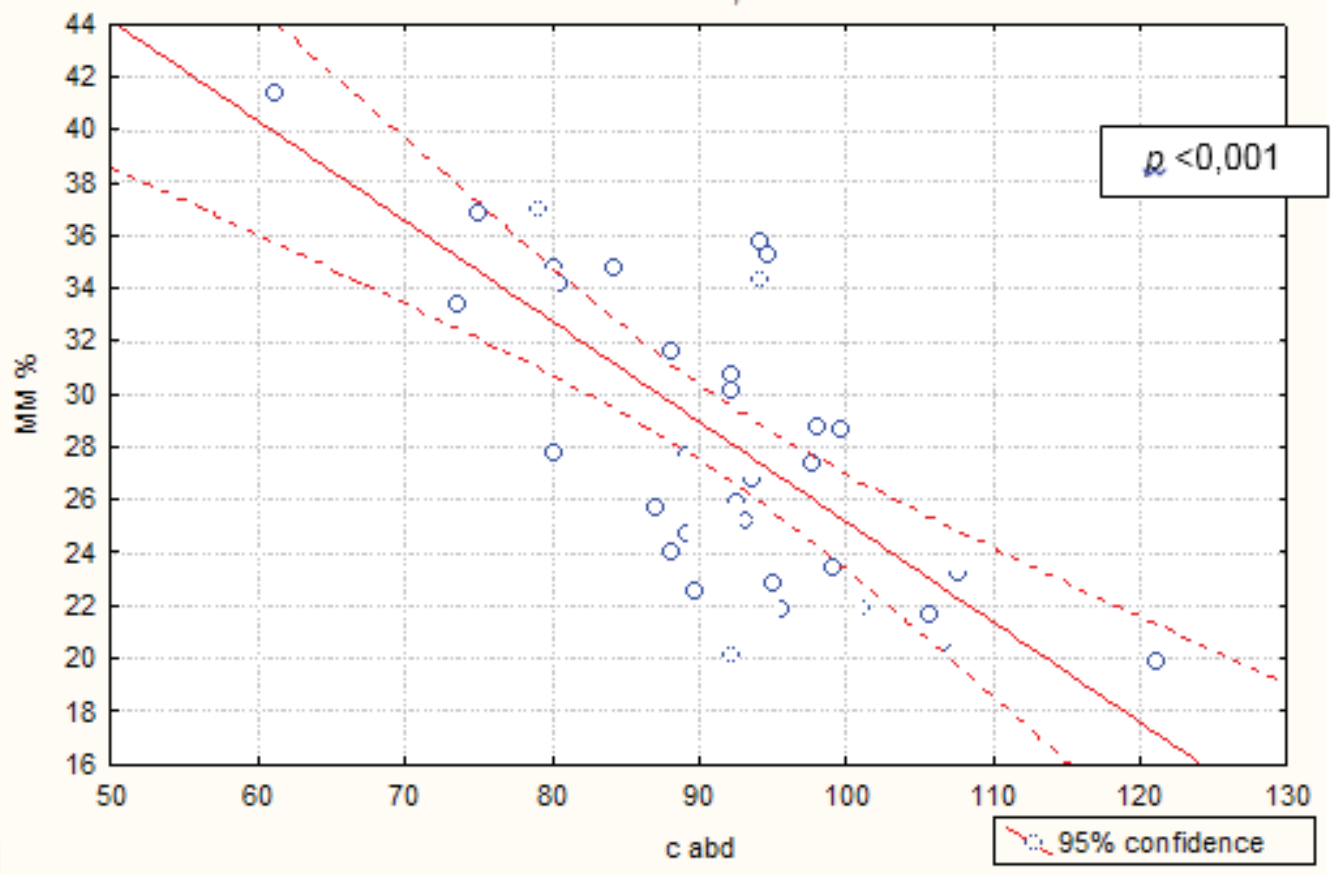

De acordo com o IMC, houve correla- ou seja, o que contribui para esse excesso de ção negativa de forma significativa com o \% MM para a amostra total e para os homens, denotando que maiores índices de massa peso é um acúmulo maior de gordura corporal, em detrimento à massa muscular. Os corporal se relacionam com menor \% MM,

Tabela 7 - Correlação entre índice de massa corporal e variáveis referentes à massa muscular.

\begin{tabular}{l|c|c|c}
\hline $\begin{array}{l}\text { Índice de massa } \\
\text { corporal }\end{array}$ & MM (Kg) & $\% \mathrm{MM}$ & $\mathrm{IMM}\left(\mathrm{Kg} / \mathrm{m}^{2}\right)$ \\
\hline Geral & $\mathrm{r}=0,24(p=0,10)$ & $\mathrm{r}=-0,54(p=0,001)^{\star}$ & $\mathrm{r}=0,32(p=0,028)^{\star}$ \\
\hline Homens & $\mathrm{r}=0,35(p=0,039)^{\star}$ & $\mathrm{r}=-0,56(p=0,000)^{\star}$ & $\mathrm{r}=0,38(p=0,26)$ \\
\hline Mulheres & $\mathrm{r}=0,71(p=0,01)^{\star}$ & $\mathrm{r}=-0,54(p=0,07)$ & $\mathrm{r}=0,51(p=0,087)$ \\
\hline
\end{tabular}

r: coeficiente de correlação linear. ${ }^{\star}$ Valor de $p$ significativo, $<0,05$. 
Os resultados foram significativos entre ambos os gêneros para IMC e MM (Kg), haja vista que essa unidade de medida está condicionada ao peso corporal, esses indivíduos podem apresentar maior quantidade de musculatura, inclusive devido à infiltração adiposa.

\section{Discussão}

A avaliação do estado nutricional, sobretudo a análise de massa muscular e sua relação com a adiposidade corporal, é de extrema significância, visto que uma quantificação detalhada de cada um dos elementos que compõem essas análises atrelado à evidências científicas a respeito do diagnóstico de anormalidades corporais, possibilita avaliar o grau de risco, principalmente para o desenvolvimento de doenças crônicas não transmissíveis (DCNT), prevenindo ou na medida do possível, revertendo quadros agudos evidenciados pelo desencadeamento dessas doenças.

Em avaliação de indivíduos com sobrepeso em ambulatório, foi verificado maiores números de mulheres com hipertensão arterial sistêmica (HAS), ${ }^{21}$ resultado ao encontro do presente estudo em que a HAS foi a doença de maior prevalência, sobretudo entre o gênero feminino. A relação entre hipertensão arterial e circunferência da cintura é fortemente evidenciada e explicada por Santos, Araújo e Cabral, ${ }^{21}$ ao apontar que $85 \%$ das mulheres tinham excesso de peso, sendo que desse total, $81 \%$ apresentavam circunferência da cintura muito elevada e possivelmente esse cenário condicionou a maioria delas à hipertensão arterial verificada. O componente familiar se expressa fortemente quando não levado em consideração o cuidado antecipado, conforme mostrado com o presente estudo, demonstrando a existência de um impacto dos antecedentes maternos sobre o desenvolvimento de HAS em mulheres $(57,14 \%)$ e dos antecedentes paternos como influência ao desenvolvimento da HAS em homens $(66,66 \%)$.

Deve-se buscar empregar indicadores mais representativos de desordens metabólicas, com destaque para a circunferência da cintura e o índice de massa muscular. ${ }^{22}$ Acerca da circunferência da cintura, a média obtida para os homens $(101,25 \mathrm{~cm})$ foi maior que a circunferência das mulheres $(91,17$ $\mathrm{cm}$ ), entretanto, a classificação dos homens seria dada como circunferência abdominal aumentada, embora próximo ao limítrofe dessa classificação, ao passo que para as mulheres, esse valor já se enquadra como muito aumentado. Essas médias foram similares a outro estudo de avaliação antropométrica, ${ }^{22}$ cujo valor médio da CC foi de 99,4 cm e 88,8 $\mathrm{cm}$ para os sexos masculino e feminino na devida ordem e a classificação do risco se deu da mesma forma aos pontos de corte adotados neste estudo.

$\mathrm{O}$ índice de massa corporal (IMC) e circunferência da cintura (CC) são fatores preditivos para comorbidades relacionadas à obesidade, mas somente a CC em análise isolada possui associação positiva e significativa. ${ }^{8}$ Os valores de IMC neste estudo em ambos os sexos apresentaram-se dentro do intervalo de corte para sobrepeso, embora a média de $29,52 \pm 5,15 \mathrm{Kg} / \mathrm{m}^{2}$ tenha sido muito próxima ao corte mínimo para obesidade grau $\mathrm{I}$, sobretudo entre as mulheres $29,85 \pm 5,31 \mathrm{Kg} / \mathrm{m}^{2}$. Ainda associando o valor médio de IMC das pacientes com a CC 
obtida, pode-se considerar que esse IMC com tendência à obesidade especialmente para esse gênero, é decorrente de um predomínio adiposo padrão androide.

A média do índice de massa muscular (IMM) no grupo masculino evidenciou sarcopenia grau $\mathrm{I}\left(10,04 \mathrm{Kg} / \mathrm{m}^{2}\right)$, enquanto que a média das mulheres não indicou comprometimento muscular (Tabela 1). A sarcopenia é significativamente maior para homens do que para mulheres, em todas as idades. Conforme estudo de avaliação de sarcopenia, o risco para sarcopenia acometer homens foi 9,84 vezes maior, e $27 \%$ foram diagnosticados sarcopênicos, o que não foi observado em mulheres. ${ }^{3}$ Em análise específica de musculatura, Janssen et al. ${ }^{8}$ encontraram em sua amostra uma média de IMM de $7,04 \mathrm{Kg} / \mathrm{m}^{2}$ para mulheres e $9,86 \mathrm{Kg} / \mathrm{m}^{2}$ para os homens, um resultado muito semelhante ao encontrado na amostra deste estudo, cujos valores foram $8,34 \mathrm{Kg} / \mathrm{m}^{2}$ e $10,04 \mathrm{Kg} / \mathrm{m}^{2}$, respectivamente. $\mathrm{O}$ fato é que os homens não possuem uma proteção hormonal, assim começam a perder massa muscular mais precocemente do que as mulheres. ${ }^{23,24}$

A prevalência de sarcopenia é variável a depender do critério diagnóstico e pontos de corte adotados. Alguns estudos identificaram $12,4 \%$ das mulheres entre 45 65 anos de idade com sarcopenia, ${ }^{25}$ enquanto Manda e Burini, ${ }^{26}$ utilizando o mesmo ponto de corte ${ }^{8}$ que o presente estudo, verificaram sarcopenia em $15,25 \%$ das mulheres e $65,26 \%$ dos homens, ou seja, uma proporção muito maior em homens, evidenciando resultados bastante similares com o estudo atual (66,67\% homens e $11,43 \%$ mulheres). Entretanto, demais pesquisadores ${ }^{27}$ chegaram a resultados diferentes, em que $11,8 \%$ das mulheres eram sarcopênicas frente a 9,7\% dos homens maiores de 20 anos de idade em estudo com a população coreana.

Em estudo da sarcopenia estratificada para sexo e faixas etárias, ${ }^{19}$ foi descoberto que na idade de 18-29 anos $14 \%$ das mulheres já apresentavam sarcopenia grau I, provavelmente devido ao desuso muscular, uma vez que nessa faixa etária ainda prevalece o pico muscular que começa a declinar mais acentuadamente após os 50 anos de idade. Na faixa etária de 30-39 anos, $2 \%$ tinham sarcopenia grau II. Os dados enfatizam a susceptibilidade masculina ao decréscimo muscular, ao verificarem que, na faixa de 18-29 anos de idade, $10 \%$ eram sarcopênicos grau I e $2 \%$ grau II, sendo que o grau II nas mulheres apareceu somente em idades posteriores. Esses dados estão de acordo com os resultados encontrados em que, justamente, a média de idade de homens sarcopênicos foi de 28,5 $\pm 3,85$ anos (Tabela 4).

Considerando a amostra total, $80,86 \%$ apresentaram determinada anormalidade para a circunferência da cintura, um resultado bastante preocupante na visão clínica, tendo em vista as associações de outras alterações metabólicas que podem suceder a esse diagnóstico senão serem a causa dessa obesidade central. ${ }^{1,3}$ Em análise dos indicadores antropométricos para risco de anormalidades metabólicas, Mota et al. ${ }^{22}$ verificaram que, independentemente do estado nutricional (sobrepeso e obesidade) e gênero, aqueles com a circunferência da cintura alterada apresentaram maiores concentrações de glicemia de jejum, triglicerídeos, colesterol total, LDL-col e menor HDL-col em relação aos eutróficos. Nesse caso, evidencia-se que o excesso de peso, indepen- 
dentemente do grau, é capaz de promover um comprometimento significativo desses marcadores bioquímicos que, inclusive, fazem parte do diagnóstico para a síndrome metabólica. ${ }^{28}$

Conforme abordado na Tabela 6, houve correlação negativa entre CC e \% MM em todos os grupos, bem como, IMC e \% MM (Tabela 7) para o grupo geral e homens, significando que são inversamente proporcionais, assim, em excesso de peso a massa muscular está comprometida, havendo um desequilíbrio na composição corporal. Há evidências que, quanto maior a distribuição de gordura na região abdominal verificada pela CC, tanto menor quantidade de massa muscular os indivíduos apresentavam, uma vez que esse valor se dá com base no peso corporal total e se há um excesso adiposo, por conseguinte, o percentual de massa muscular referente ao peso total será inferior.

Estudos verificaram a existência de uma associação positiva entre IMC e CC com o IMM, ${ }^{3,29}$ especialmente naqueles indivíduos que apresentam concentração adiposa na região abdominal. De igual modo, quando majoritariamente a distribuição adiposa é central, a musculatura de membros inferiores e superiores pode estar comprometida, em virtude do desuso ou menor mobilização por completa da capacidade muscular, conforme abordado em teste de suporte para cadeiras, no qual se avaliou o tempo para sentar e levantar, ${ }^{25}$ o grupo de mulheres obe- sas e obesas sarcopênicas obteve um tempo maior, em razão do fato de que o tempo de deslocamento do corpo está atrelado à força que, por sua vez, é condicionada pela potência muscular e coordenação. A perda de força pode até ser maior do que o prejuízo de perda de massa, uma vez que ela depende do grau de utilização muscular, logo, elevadas quantidades de massa muscular podem estar sujeitas à não funcionalidade.

\section{Considerações finaIs}

Em face aos resultados apresentados, a partir da análise de sarcopenia, concluiu-se que há perda muscular mais evidente entre o gênero masculino. Em ambos os gêneros, foi verificado excesso de peso em algum grau, sobretudo em mulheres, que evidencia um predomínio de gordura abdominal. Os homens podem apresentar maior susceptibilidade para as consequências da obesidade sarcopênica em razão do excesso de peso e sarcopenia identificados simultaneamente. Apesar dos valores dentro da normalidade para massa muscular entre a maioria das mulheres, não há como desconsiderar uma possibilidade para obesidade sarcopênica, pois embora não detectada antropometricamente, de forma mais sensível como os homens, o grau de utilização e eficácia muscular correlaciona-se inversamente com a quantidade de massa gorda. 


\section{REFERÊNCIAS}

1. Manda RM. Obesidade Sarcopênica: diagnóstico, prevalência e associações com aptidão física, resistência insulínica, estresse inflamatório e oxidativo. [Dissertação]. Botucatu (SP): Universidade Estadual Paulista "Júlio de Mesquita Filho"; 2013.

2. Kim TN, Choi KM. The implications of sarcopenia and sarcopenic obesity on cardiometabolic disease. J Cell Biochem. [Internet], 2015; 116 (7): 1.171-8.

3. Pierine DT. Associação da massa muscular esquelética com variáveis demográficas, antropométricas, dietéticas, bioquímicas e aptidão física de adultos clinicamente selecionados para programa de mudança de estilo de vida (MEV). [Dissertação]. Botucatu (SP): Universidade Estadual Paulista "Júlio de Mesquita Filho"; 2010.

4. Roubenoff R. Sarcopenic obesity: does muscle loss cause fat gain? Lessons from rheumatoid arthritis and osteoarthritis. Ann N Y Acad Sci. [Internet], 2000; 904 (1): 553-7.

5. Srikanthan P., Hevener AL, Karlamangla AS. Sarcopenia exacerbates obesity- Associated insulin resistance and dysglycemia: findings from the National Health and Nutrition Examination Survey III. PloS One. [Internet], 2010; 5 (5): 1-7.

6. Kim J-H, Cho JJ, Park YS. Relationship between sarcopenic obesity and cardiovascular disease risk as estimated by the Framingham Risk Score. J Korean Med Sci. [Internet], 2015; 30 (3): 264-71.

7. Park SH, Park JH, Park HY, Jang HJ, Kim HK, Park J., et al. Additional role of sarcopenia to waist circumference in predicting the odds of metabolic syndrome. Clin Nutr. [Internet], 2014; 33 (4): 668-72.

8. Janssen I., Baumgartner RN, Ross R., Rosenberg IH, Roubenoff R. Skeletal muscle cutpoints associated with elevated physical disability risk in older men and women. Am J Epidemiol. [Internet], 2004; 159 (4): 413-21.

9. International Physical Activity Questionnaire (IPAQ). Guidelines for data processing and analysis of the International Physical Activity Questionnaire (IPAQ) - short and long forms, 2004 [Acesso em 8 de março de 2017]. Disponível em: http://www.institutferran.org/ documentos/scoring_short_ipaq_april04.pdf.

10. World Health Organization. For cutting down or stopping substance use-A guide. Self-Help Strategies. France, 2010 [Acesso em 8 de março de 2017]. Disponível em: http://apps.who.int/ iris/bitstream/10665/44322/1/9789241599405_eng.pdf

11. Heyward VH, Stolarczyk LM. Avaliação da composição corporal aplicada, 1. ed. Barueri (SP): Manole; 2000.

12. Frisancho, AR. Anthropometric Standards for the Assessment of Growth and Nutritional Status, 1. ed. Ann Arbor (Michigan): University of Michigan Press; 1990.

13. Lohman T., Roche AF, Martorell R. Anthropometric Standardization Reference Manual, 1. ed. Champaign (Illinois): Human Kinetics books; 1988.

14. World Health Organization. Report of a WHO Consultation. Obesity: Preventing and managing the global epidemic. Geneva (Swissland): WHO/FAO, 2000 [Acesso em 22 de janeiro de 2017]. Disponível em: file://C:/Users/User/Downloads/WHO_TRS_894.pdf.

15. Federação Latino-americana de Sociedades de Obesidade - FLASO. Consenso Latino-Americano de Obesidade. Arq Bras Endocrinol Metab. [Internet], 1999; 43 (1): 21-67.

16. Rolland Y., Lauwers-Cances V., Cournot M., Nourhashémi F., Reynish W., Rivière D., et al. Sarcopenia, calf circumference, and physical function of elderly women: a cross-sectional study. Journal American Geriatrics Society. [Internet], 2003; 51 (8): 1.120-4.

17. Frisancho AR. New norms of upper limb fat and muscle areas for assessment of nutritional status. Am J Cli Nutr. [Internet], 1981; 34 (11): 2.540-5.

18. Lee RC, Wang Z., Heo M., Ross R., Janssen I., Heymsfield SB. Total-body skeletal muscle mass: development and cross-validation of anthropometric prediction models. Am J Cli Nutr. [Internet], 2000; 72 (3): 796-803. Errata em: Am J Cli Nutr. [Internet], 2001; 73 (5): 995. 
19. Janssen I., Heymsfield SB, Ross R. Low relative skeletal muscle mass (sarcopenia) in older persons is associated with functional impairment and physical disability. J Am Geriatr Soc. [Internet], 2002; 50 (5): 889-96.

20. Baumgartner RN, Koehler KM, Gallagher D., Romero L., Heymsfield SB, Ross RR, et al. Epidemiology of Sarcopenia among the Elderly in New México. Am J Epidemiol. [Internet], 1998; 147 (8): 755-63. Errata em: Am J Epidemiol. [Internet], 1999; 149 (12): 1.161.

21. Santos CM, Araújo AR, Cabral PC. Avaliação antropométrica de pacientes com sobrepeso atendidos em ambulatórios de nutrição. Revista da Associação Brasileira de Nutrição- ASBRAN. [Internet], 2013; 5 (1): 13-20.

22. Mota JF, Rinaldi AEM, Pereira AF, Orsatti FL, Burini RC. Indicadores antropométricos como marcadores de risco para anormalidades metabólicas. Ciênc. Saúde Coletiva. [Internet], 2011; 16 (9): 3.901-8.

23. Janssen I., Heymsfield SB, Baumgartner RN, Ross R. Estimation of skeletal muscle mass by bioelectrical impedance analysis. J Appl Physiol. [Internet], 2000; 89 (2): 465-71.

24. Lee JSW, Auyeung TW, Kwok T., Lau EM, Leung PC, Woo J. Associated Factors and Health Impact of Sarcopenia in Older Chinese Men and Women: A Cross-Sectional Study. Gerontology. [Internet], 2007; 53 (6): 404-10.

25. Moreira MA, Zunzunegui MV, Vafaei A., Câmara SM, Oliveira TS, Maciel ÁC. Sarcopenic obesity and physical performance in middle aged women: a cross-sectional study in Northeast Brazil. BMC Public Health. [Internet], 2.016; 16 (43): 2-10.

26. Manda RM, Burini RC. Alterações sanguíneas, da capacidade cardiorrespiratória e aptidão física em adultos portadores de Obesidade Sarcopênica. [Dissertação]. Botucatu (SP): Universidade Estadual Paulista "Júlio de Mesquita Filho"; 2013.

27. Kim YS, Lee Y., Chung YS, Lee DJ, Joo NS, Hong D., et al. Prevalence of Sarcopenia and Sarcopenic Obesity in the Korean Population Based on the Fourth Korean National Health and Nutritional Examination Surveys. J Gerontol A Biol Sci Med Sci. [Internet], 2012; 67 (10): 1.107-13.

28. $7^{\circ}$. Diretriz Brasileira de Hipertensão Arterial. Arq Bras Cardiol. [Internet], 2016; 107 (3 Suppl 3): 1-83.

29. Cruz-Jentoft AJ, Baeyens JP, Bauer JM, Boirie Y., Cederholm T., Landi F., et al. Sarcopenia: European Consensus on Definition and Diagnosis- Report of the European Working Group on Sarcopenia in Older People. Age Ageing. [Internet], 2010; 39 (4): 412-23.

\section{Dados das autoras}

\section{Beatriz Cassaniga Talassi}

Graduada em Nutrição pela Universidade Metodista de Piracicaba (UNIMEP). Nutricionista. Piracicaba/SP-Brasil. beatriztalassi@yahoo.com.br

\section{Amanda Martim}

Graduanda em Nutrição pela Universidade Metodista de Piracicaba (UNIMEP). Nutricionista. Piracicaba/SP-Brasil. amandamartim@outlook.com.br

\section{Iza Oliveira Hoff}

Mestre em saúde da criança e do adolescente pela Universidade Estadual de Campinas (UNICAMP). Docente na Universidade Metodista de Piracicaba (UNIMEP). Piracicaba/SP-Brasil. iza.o.hoff@ gmail.com

Submetido em: 17-4-2018

Aceito em: 3-10-2018 